Tôhoku Math. Journ.

30 (1978), 543-551.

\title{
$\sigma$-HYPERSURFACES IN A LOCALLY SYMMETRIC ALMOST HERMITIAN MANIFOLD
}

\author{
Sumio Sawaki and Kouei Sekigawa
}

(Received April 25, 1977, revised June 3, 1977)

0. Introduction. Let $\widetilde{M}^{n+p}$ be an $n+p$-dimensional $C^{\infty}$ Riemannian manifold with metric tensor $\widetilde{g}$ and Levi-Civita connection $\tilde{\nabla}$. Then the curvature tensor $\widetilde{R}$ of $\widetilde{M}^{n+p}$ is given by $\widetilde{R}(X, Y)=\left[\tilde{V}_{X}, \tilde{V}_{Y}\right]-\tilde{V}_{[X, Y]}$ for any $X, Y \in \mathfrak{X}(\tilde{M})$ where $\mathfrak{X}(\tilde{M})$ is the Lie algebra of $C^{\infty}$ vector fields in $\tilde{M}^{n+p}$.

Moreover, let $M^{n}$ be an $n$-dimensional submanifold immersed in $\tilde{M}^{n+p}$. Then we have

$$
\tilde{\nabla}_{X} Y=\nabla_{X} Y+\sigma(X, Y) \quad \text { for any } \quad X, Y \in \mathfrak{X}(M)
$$

where $\nabla_{X} Y$ and $\sigma$ denote the component of $\tilde{\nabla}_{X} Y$ tangent to $M^{n}$ and the second fundamental form of $M^{n}$ in $\tilde{M}^{n+p}$, respectively. It is well known that $\nabla$ is the covariant differentiation of $M^{n}$ and $\sigma$ is a symmetric covariant tensor field of degree 2 with values in the normal bundle $T(M)^{\perp}$ of $M^{n}$ where $T(M)$ denotes the tangent bundle of $M^{n}$.

We have further

$$
\tilde{\nabla}_{X} \xi_{\alpha}=-A_{\alpha} X+\sum_{\beta=1}^{p} s_{\alpha \beta}(X) \xi_{\beta} \quad(\alpha=1,2, \cdots, p)
$$

where $\left\{\xi_{\alpha}\right\}$ is a local orthonormal frame field for $T(M)$ and $-A_{\alpha} X$ is the tangential component of $\tilde{\nabla}_{X} \xi_{\alpha}$.

Let $\nabla^{\prime}$ be the covariant differentiation with respect to the connection in $T(M) \oplus T(M)^{\perp}$. Then we have

$$
\left(\nabla_{X}^{\prime} \sigma\right)(Y, Z)=\left(\tilde{\nabla}_{X} \sigma(Y, Z)\right)^{\perp}-\sigma\left(\nabla_{X} Y, Z\right)-\sigma\left(Y, \nabla_{X} Z\right)
$$

for any $X, Y, Z \in \mathfrak{X}(M)$.

If $\nabla_{X}^{\prime} \sigma=0$ for any $X \in \mathfrak{X}(M)$, then the second fundamental form is said to be parallel. $M^{n}$ is said to be curvature invariant if $\widetilde{R}(X, Y) Z$ belongs to $\mathfrak{X}(M)$ for any $X, Y, Z \in \mathfrak{X}(M)$.

Next, let $\widetilde{M}^{2 m+2 q}$ be a $2 m+2 q$-dimensional almost Hermitian manifold with an almost Hermitian structure $(\widetilde{J}, \widetilde{g})$. Then a $2 m$-dimensional invariant submanifold $M^{2 m}$ of $\widetilde{M}^{2 m+2 q}$ is said to be a $\sigma$-submanifold if the second fundamental form $\sigma$ is complex bilinear, i.e., 


$$
\sigma(J X, Y)=\sigma(X, J Y)=\widetilde{J} \sigma(X, Y) \quad \text { for any } \quad X, Y \in \mathfrak{X}(M),
$$

where $J$ is the induced almost complex structure on $M^{2 m}$. In particular, if $M^{2 m}$ is a $\sigma$-hypersurface of $\tilde{M}^{2 m+2}$, then the condition (0.4) is equivalent to $B=J A$ and $A J=-J A$, where $A$ and $B$ are the second fundamental tensors with respect to any unit normal vectors $\xi$ and $\widetilde{J} \xi$ to $M^{2 m}$, respectively.

An almost Hermitian manifold $\tilde{M}$ is called an ${ }^{*} O$-space (or quasiKähler manifold) [2] if

$$
\left(\tilde{\nabla}_{X} \widetilde{J}\right) Y+\left(\tilde{V}_{\tilde{J} X} \widetilde{J}\right) \widetilde{J} Y=0
$$

for any $X, Y \in \mathfrak{X}(\tilde{M})$ and $\tilde{M}$ is called a $K$-space (or Tachibana space or nearly Kähler manifold) if

$$
\left.\left(\tilde{V}_{X} \widetilde{J}\right) Y+\left(\tilde{V}_{Y} \widetilde{J}\right) X=0 \quad \text { (or equivalently }\left(\tilde{V}_{X} \widetilde{J}\right) X=0\right)
$$

for any $X, Y \in \mathfrak{X}(\tilde{M})$.

It is well known that a Kähler manifold is a $K$-space and a $K$-space is an ${ }^{*} O$-space. It is also well known that an invariant hypersurface of a $K$-space or an ${ }^{*} O$-space is a $K$-space or an ${ }^{*} O$-space respectively. Moreover, we know that an invariant hypersurface of a Kähler manifold or a $K$-space is a $\sigma$-hypersurface (see for example [3]).

The following theorem is well known.

Theorem A (B. Smyth [4], T. Takahashi [5]). Let $M^{2 m}$ be an invariant hypersurface of a Kähler manifold $\widetilde{M}^{2 m+2}$ of constant holomorphic sectional curvature. If $M^{2 m}$ is an Einstein (or Ricci parallel) manifold, then $M^{2 m}$ is locally symmetric.

A Kähler manifold $\tilde{M}^{2 m+2}$ of constant holomorphic sectional curvature is locally symmetric and its invariant hypersurface $M^{2 m}$ is curvature invariant (see for example [4]). What will become of this theorem if we replace the assumption of being of constant holomorphic sectional curvature by being locally symmetric? Our main result is

THEOREM. Let $M^{2 m}$ be a $\sigma$-hypersurface of a locally symmetric *Ospace $\widetilde{M}^{2 m+2}$. If $M^{2 m}$ is Ricci parallel and curvature invariant, then $M^{2 m}$ is locally symmetric.

COROLlaRY. Let $M^{2 m}$ be an invariant hypersurface of a locally symmetric Kähler manifold $\tilde{M}^{2 m+2}$. If $M^{2 m}$ is Ricci parallel and curvature invariant, then $M^{2 m}$ is locally symmetric.

This generalizes Theorem A. 
1. Submanifolds of a Riemannian manifold. Let $M^{n}$ be a submanifold immersed in a Riemannian manifold $\widetilde{M}^{n+p}$ and put

$$
\sigma(X, Y)=\sum_{\alpha=1}^{p} h_{\alpha}(X, Y) \xi_{\alpha}
$$

Then we have

$$
\widetilde{g}\left(\sigma(X, Y), \xi_{\alpha}\right)=g\left(A_{\alpha} X, Y\right)=g\left(X, A_{\alpha} Y\right)=h_{\alpha}(X, Y)
$$

for any $X, Y \in \mathfrak{X}(M)$ and $\xi_{\alpha} \in \mathfrak{X}(M)^{\perp}$ where $g$ denotes the induced metric tensor on $M^{n}$.

The following two lemmas are well known (see for example [1]).

LEMMA 1.1. Let $\widetilde{R}$ and $R$ be the curvature tensors of $\widetilde{M}^{n+p}$ and $M^{n}$ respectively. Then we have

$$
\begin{aligned}
& \widetilde{R}(X, Y) W=R(X, Y) W-\sum_{\alpha=1}^{p} h_{\alpha}(Y, W) A_{\alpha} X+\sum_{\alpha=1}^{p} h_{\alpha}(X, W) A_{\alpha} Y \\
&+\sum_{\alpha=1}^{p}\left[\left(\nabla_{X} h_{\alpha}\right)(Y, W)-\left(\nabla_{Y} h_{\alpha}\right)(X, W)+\sum_{\beta=1}^{p} h_{\beta}(Y, W) s_{\beta \alpha}(X)\right. \\
&\left.-\sum_{\beta=1}^{p} h_{\beta}(X, W) s_{\beta \alpha}(Y)\right] \xi_{\alpha}, \\
& s_{\alpha \beta}(X)+s_{\beta \alpha}(X)=0 \quad(\alpha, \beta=1,2, \cdots, p)
\end{aligned}
$$

for any $X, Y, W \in \mathfrak{X}(M)$.

LEMMA 1.2. $\nabla_{X}^{\prime} \sigma=0$ is equivalent to $\nabla_{X} A_{\alpha}=\sum_{\beta=1}^{p} s_{\alpha \beta}(X) A_{\beta}$ $(\alpha=1,2, \cdots, p)$.

The following lemma which plays an important role in proving the main theorem is also easily verified (see for example [6], p. 99, where $\nabla_{X}^{\prime} \sigma=0$ means $\left.\nabla_{d} h_{c b}^{x}=0\right)$.

LEMMA 1.3. Let $M^{n}$ be a submanifold immersed in a locally symmetric Riemannian manifold $\tilde{M}^{n+p}$. If the second fundamental form is parallel, then $M^{n}$ is locally symmetric.

2. Invariant hypersurfaces of an almost Hermitian manifold. Let $M^{2 m}$ be an invariant hypersurface of an almost Hermitian manifold $\tilde{M}^{2 m+2}$. Then putting

$$
\begin{array}{r}
A=A_{1}, B=A_{2}, \xi=\xi_{1}, J \xi=\xi_{2}, h(X, Y)=h_{1}(X, Y), \\
k(X, Y)=h_{2}(X, Y), s(X)=s_{12}(X), t(X)=s_{21}(X) \\
\text { for any } X, Y \in \mathfrak{X}(M)
\end{array}
$$

and rewriting (0.1), (0.2) and (1.4), we have

$$
\tilde{\nabla}_{X} Y=\nabla_{X} Y+h(X, Y) \xi+k(X, Y) \widetilde{J} \xi \text {, }
$$




$$
\begin{gathered}
\tilde{\nabla}_{X} \xi=-A X+s(X) \widetilde{J} \xi, \quad \tilde{\nabla}_{X}(\widetilde{J} \xi)=-B X+t(X) \xi, \\
s(X)+t(X)=0,
\end{gathered}
$$

respectively. Here $A$ and $B$ are symmetric tensors with respect to $g$ and from (1.2), we have

$$
h(X, Y)=g(A X, Y), \quad k(X, Y)=g(B X, Y) \text {. }
$$

Moreover, the equation (1.3) becomes

$$
\begin{aligned}
\widetilde{R}(X, Y) W=R(X, Y) W-[h(Y, W) A X-h(X, W) A Y] \\
\quad-[k(Y, W) B X-k(X, W) B Y]+\left[\left(\nabla_{X} h\right)(Y, W)-\left(\nabla_{Y} h\right)(X, W)\right. \\
\quad+k(Y, W) t(X)-k(X, W) t(Y)] \xi+\left[\left(\nabla_{X} k\right)(Y, W)\right. \\
\left.\quad-\left(\nabla_{Y} k\right)(X, W)+h(Y, W) s(X)-h(X, W) s(Y)\right] \widetilde{J} \xi
\end{aligned}
$$

From (2.6) the following well known lemma follows.

LEMMA 2.1. Let $M^{2 m}$ be an invariant hypersurface of an almost Hermitian manifold $\widetilde{M}^{2 m+2}$. If $M^{2 m}$ is curvature invariant, then we have

$$
\begin{aligned}
& \widetilde{R}(X, Y) W=R(X, Y) W-[g(A Y, W) A X-g(A X, W) A Y] \\
&-[g(B Y, W) B X-g(B X, W) B Y], \\
&\left(\nabla_{X} A\right) Y-\left(\nabla_{Y} A\right) X-s(X) B Y+s(Y) B X=0, \\
&\left(\nabla_{X} B\right) Y-\left(\nabla_{Y} B\right) X+s(X) A Y-s(Y) A X=0 \quad \text { (Codazzi equation) }
\end{aligned}
$$

for any $X, Y, W \in \mathfrak{X}(M)$.

For an almost Hermitian manifold $\widetilde{M}^{2 m+2}$, the Ricci tensor $\widetilde{S}(Y, W)$ is given by

$$
\widetilde{S}(Y, W)=\sum_{i=1}^{m} \widetilde{g}\left(\widetilde{R}\left(e_{i}, Y\right) W, e_{i}\right)+\sum_{i=1}^{m} \widetilde{g}\left(\widetilde{R}\left(J e_{i}, Y\right) W, J e_{i}\right)+L(Y, W)
$$

for any $Y, W \in \mathfrak{X}(M)$, where $L(Y, W)=\widetilde{g}(\widetilde{R}(\xi, Y) W, \xi)+\widetilde{g}(\widetilde{R}(\widetilde{J} \xi, Y) W$, $\widetilde{J} \xi)$ and $\left\{e_{1}, \cdots, e_{m}, J e_{1}, \cdots, J e_{m}\right\}$ is an orthonormal frame field defined on an open set $U$ of $M^{2 m}$. It is easily seen that $L(Y, W)$ is a symmetric tensor field of type $(0,2)$ on $M^{2 m}$.

LEMмA 2.2. Let $M^{2 m}$ be an invariant hypersurface of a locally symmetric almost Hermitian manifold $\tilde{M}^{2 m+2}$. If $M^{2 m}$ is curvature invariant, then we have

$$
\begin{gathered}
\left(\nabla_{X} L\right)(Y, W)=k(X, Y) \widetilde{S}(\widetilde{J} \xi, W)+k(X, W) \widetilde{S}(\widetilde{J} \xi, Y) \\
+h(X, Y) \widetilde{S}(\xi, W)+h(X, W) \widetilde{S}(\xi, Y)
\end{gathered}
$$

for any $X, Y, W \in \mathfrak{X}(M)$. 
Proof. We have

$$
\left(\nabla_{X} L\right)(Y, W)=X(L(Y, W))-L\left(\nabla_{X} Y, W\right)-L\left(Y, \nabla_{X} W\right) .
$$

For the first term of the right hand side of (2.12), we have

$$
X(L(Y, W))=X(\widetilde{g}(\widetilde{R}(\xi, Y) W, \xi))+X(\widetilde{g}(\widetilde{R}(\widetilde{J} \xi, Y) W, \widetilde{J} \xi)) .
$$

Since $M^{2 m}$ is curvature invariant, we have

$$
\widetilde{g}(\widetilde{R}(A X, Y) W, \xi)=0, \quad \widetilde{g}(\widetilde{R}(\xi, Y) W, A X)=-\widetilde{g}(\widetilde{R}(W, A X) Y, \xi)=0 .
$$

Hence, making use of (2.2) and (2.3), we have

$$
\begin{aligned}
X(\widetilde{g}(\widetilde{R}(\xi, Y) W, \xi)) & =s(X) \widetilde{g}(\widetilde{R}(\widetilde{J} \xi, Y) W, \xi)+\widetilde{g}\left(\widetilde{R}\left(\xi, \nabla_{X} Y\right) W, \xi\right) \\
& +k(X, Y) \widetilde{g}(\widetilde{R}(\xi, \widetilde{J} \xi) W, \xi)+\widetilde{g}\left(\widetilde{R}(\xi, Y) V_{X} W, \xi\right) \\
& +k(X, W) \widetilde{g}(\widetilde{R}(\xi, Y) \widetilde{J} \xi, \xi)+s(X) \widetilde{g}(\widetilde{R}(\xi, Y) W, \widetilde{J} \xi) .
\end{aligned}
$$

For the third term of the right hand side of (2.14), making use of $\widetilde{g}\left(\widetilde{R}\left(e_{i}, \widetilde{J} \xi\right) W, e_{i}\right)=0$ and $\widetilde{g}\left(\widetilde{R}\left(J e_{i}, \widetilde{J} \xi\right) W, J e_{i}\right)=0$, we have

$$
\begin{aligned}
& k(X, Y) \widetilde{g}(\widetilde{R}(\xi, \widetilde{J} \xi) W, \xi)=k(X, Y)\left[\sum_{i=1}^{m} \widetilde{g}\left(\widetilde{R}\left(e_{i}, \widetilde{J} \xi\right) W, e_{i}\right)\right. \\
& \left.\quad+\sum_{i=1}^{m} \widetilde{g}\left(\widetilde{R}\left(J e_{i}, \widetilde{J} \xi\right) W, J e_{i}\right)+\widetilde{g}(\widetilde{R}(\xi, \widetilde{J} \xi) W, \xi)+\widetilde{g}(\widetilde{R}(\widetilde{J} \xi, \widetilde{J} \xi) W, \widetilde{J} \xi)\right] \\
& =k(X, Y) \widetilde{S}(\widetilde{J} \xi, W) .
\end{aligned}
$$

Similarly, for the fifth term, we have

$$
k(X, W) \widetilde{g}(\widetilde{R}(\xi, Y) \widetilde{J} \xi, \xi)=k(X, W) \widetilde{S}(\widetilde{J} \xi, Y) .
$$

Thus, (2.14) turns out to be

(2.15) $X(\widetilde{g}(\widetilde{R}(\xi, Y) W, \xi))$

$$
\begin{aligned}
= & s(X)[\widetilde{g}(\widetilde{R}(\widetilde{J} \xi, Y) W, \xi)+\widetilde{g}(\widetilde{R}(\xi, Y) W, \widetilde{J} \xi)]+\widetilde{g}\left(\widetilde{R}\left(\xi, \nabla_{X} Y\right) W, \xi\right) \\
& +\widetilde{g}\left(\widetilde{R}(\xi, Y) \nabla_{X} W, \xi\right)+k(X, Y) \widetilde{S}(\widetilde{J} \xi, W)+k(X, W) \widetilde{S}(\widetilde{J} \xi, Y) .
\end{aligned}
$$

Similarly, for the second term of the right hand side of (2.13), we have

$$
\begin{aligned}
X(\widetilde{g}(\widetilde{R}(\widetilde{J} \xi, Y) W, \widetilde{J} \xi)) & =-s(X)[\widetilde{g}(\widetilde{R}(\xi, Y) W, \widetilde{J} \xi)+\widetilde{g}(\widetilde{R}(\widetilde{J} \xi, Y) W, \xi)] \\
& +\widetilde{g}\left(\widetilde{R}\left(\widetilde{J} \xi, \nabla_{X} Y\right) W, \widetilde{J} \xi\right)+\widetilde{g}\left(\widetilde{R}(\widetilde{J} \xi, Y) \nabla_{X} W, \widetilde{J} \xi\right) \\
& +h(X, Y) \widetilde{S}(\xi, W)+h(X, W) \widetilde{S}(\xi, Y) .
\end{aligned}
$$

Consequently, by (2.15) and (2.16), (2.12) turns out to be

$$
\begin{aligned}
& \left(\nabla_{X} L\right)(Y, W)=\widetilde{g}\left(\widetilde{R}\left(\xi, \nabla_{X} Y\right) W, \xi\right)+\widetilde{g}\left(\widetilde{R}(\xi, Y) \nabla_{X} W, \xi\right) \\
& \quad+\widetilde{g}\left(\widetilde{R}\left(\widetilde{J} \xi, \nabla_{X} Y\right) W, \widetilde{J} \xi\right)+\widetilde{g}\left(\widetilde{R}(\widetilde{J} \xi, Y) \nabla_{X} W, \widetilde{J} \xi\right)-L\left(\nabla_{X} Y, W\right)
\end{aligned}
$$




$$
\begin{aligned}
& -L\left(Y, \nabla_{X} W\right)+k(X, Y) \widetilde{S}(\widetilde{J} \xi, W)+k(X, W) \widetilde{S}(\widetilde{J} \xi, Y) \\
& +h(X, Y) \widetilde{S}(\xi, W)+h(X, W) \widetilde{S}(\xi, Y)=k(X, Y) \widetilde{S}(\widetilde{J} \xi, W) \\
& +k(X, W) \widetilde{S}(\widetilde{J} \xi, Y)+h(X, Y) \widetilde{S}(\xi, W)+h(X, W) \widetilde{S}(\xi, Y),
\end{aligned}
$$

because of the definition of $L(Y, W)$.

LeMma 2.3. Let $M^{2 m}$ be a curvature invariant $\sigma$-hypersurface of an ${ }^{*} O$-space $\widetilde{M}^{2 m+2}$. Then we have

$$
\begin{gathered}
\left(\nabla_{X} J\right) A Y=0, \quad A\left(\nabla_{X} J\right) Y=0, \\
\left(\nabla_{X} A\right) J Y=-J\left(\nabla_{X} A\right) Y
\end{gathered}
$$

for any $X, Y \in \mathfrak{X}(M)$.

Proof. Substituting $B=J A$ into (2.9), we have

$$
J\left(\nabla_{X} A\right) Y+\left(\nabla_{X} J\right) A Y-J\left(\nabla_{Y} A\right) X-\left(\nabla_{Y} J\right) A X+s(X) A Y-s(Y) A X=0 .
$$

Applying $-J$, we have

$$
\begin{array}{r}
\left(\nabla_{X} A\right) Y-\left(\nabla_{Y} A\right) X-s(X) J A Y+s(Y) J A X \\
-J\left[\left(\nabla_{X} J\right) A Y-\left(\nabla_{Y} J\right) A X\right]=0 .
\end{array}
$$

Comparing (2.8) with (2.19), we have

$$
\left(\nabla_{X} J\right) A Y=\left(\nabla_{Y} J\right) A X .
$$

Replacing $Y$ by $J Y$, we have

$$
\left(\nabla_{X} J\right) A J Y=\left(\nabla_{J Y} J\right) A X .
$$

Then, using $J^{2}=-I$, we have

$$
\left(\nabla_{X} J\right) J A J Y=\left(\nabla_{J Y} J\right) J A X
$$

or by $J A=-A J$

$$
\left(\nabla_{X} J\right) A Y=\left(\nabla_{J Y} J\right) J A X .
$$

Thus, forming the sum $(2.20)+(2.22)$, by $(0.5)$ we have

$$
\left(\nabla_{X} J\right) A Y=0 \quad \text { for any } X, Y \in \mathfrak{X}(M) .
$$

Since $A$ and $J$ are symmetric and skew-symmetric respectively, the other formula of (2.17) follows immediately from (2.23). For (2.18), by (2.17) and $J A=-A J$, we have

$$
\begin{aligned}
& \left(\nabla_{X} A\right) J Y=\nabla_{X}(A J Y)-A \nabla_{X}(J Y)=-\nabla_{X}(J A Y)-A \nabla_{X}(J Y) \\
& \quad=-\left(\nabla_{X} J\right) A Y-J\left(\nabla_{X} A\right) Y-J A\left(\nabla_{X} Y\right)-A\left(\nabla_{X} J\right) Y-A J\left(\nabla_{X} Y\right) \\
& \quad=-J\left(\nabla_{X} A\right) Y .
\end{aligned}
$$


3. Proof of Theorem. By Lemma 1.3, it is sufficient to show that $\nabla_{X}^{\prime} \sigma=0$ or by Lemma 1.2 ,

$$
\nabla_{X} A=s(X) J A, \quad \nabla_{X}(J A)=-s(X) A .
$$

From (2.7) it follows that the linear endomorphism of $T_{y}(M)\left(y \in M^{2 m}\right)$ determined by $X \mapsto \widetilde{R}(X, Y) W$ has the trace

$$
\operatorname{trace}(X \mapsto \widetilde{R}(X, Y) W)=S(Y, W)+2 g\left(A^{2} Y, W\right)
$$

for any $X, Y, W \in T_{y}(M)$, where $S(Y, W)$ is the Ricci tensor of $M^{2 m}$.

Thus, by (2.10), we have

$$
\widetilde{S}(Y, W)=S(Y, W)+2 g\left(A^{2} Y, W\right)+L(Y, W) .
$$

On the other hand, taking account of the fact that the Ricci tensor $\widetilde{S}$ of the locally symmetric manifold $\widetilde{M}^{2 m+2}$ is parallel, we have

$X \widetilde{S}(Y, W)$

$$
\begin{aligned}
& =\widetilde{S}\left(V_{X} Y+h(X, Y) \xi+k(X, Y) \widetilde{J} \xi, W\right)+\widetilde{S}\left(Y, \nabla_{X} W+h(X, W) \xi\right. \\
& \quad+k(X, W) \widetilde{J} \xi) \\
& =\widetilde{S}\left(V_{X} Y, W\right)+\widetilde{S}\left(Y, \nabla_{X} W\right)+h(X, Y) \widetilde{S}(\xi, W)+k(X, Y) \widetilde{S}(\widetilde{J} \xi, W) \\
& \quad+h(X, W) \widetilde{S}(Y, \xi)+k(X, W) \widetilde{S}(Y, \widetilde{J} \xi) .
\end{aligned}
$$

Consequently, operating $\nabla_{X}$ on both sides of (3.3) and making use of Lemma 2.2 and $\nabla_{X} S=0$, we have

$$
\nabla_{X} A^{2}=0 \text {. }
$$

Then, let us consider the distributions $D^{\alpha}(\alpha=1,2, \cdots, l)$ on a neighborhood $U(x)$ of each point $x \in M^{2 m}$ defined by

$$
D^{\alpha}(y)=\left\{X \in T_{y}(M) ; A^{2} X=\lambda_{\alpha}^{2} X\right\},
$$

where $\lambda_{\alpha}$ are nonnegative constant with $\lambda_{\alpha} \neq \lambda_{\beta}(\alpha \neq \beta)$ and $y \in U(x)$. By (3.4), $D^{\alpha}(\alpha=1,2, \cdots, l)$ is parallel and

$$
T_{y}(M)=D^{1}(y) \oplus \cdots \oplus D^{l}(y)
$$

on $U(x)$. Furthermore, $D^{\alpha}(\alpha=1,2, \cdots, l)$ are invariant under $J$ by virtue of $J A=-A J$ and therefore $J A^{2}=A^{2} J$.

Hence, we can take the distributions $D_{+}^{\alpha}, D_{-}^{\alpha}(\alpha=1,2, \cdots, l)$ on $U(x)$ given by

$$
\begin{aligned}
& D_{+}^{\alpha}(y)=\left\{X \in T_{y}(M) ; A X=\lambda_{\alpha} X\right\}, \\
& D_{-}^{\alpha}(y)=\left\{X \in T_{y}(M) ; A X=-\lambda_{\alpha} X\right\} .
\end{aligned}
$$

Then we have 


$$
D^{\alpha}(y)=D_{+}^{\alpha}(y) \oplus D_{-}^{\alpha}(y), D_{-}^{\alpha}(y)=J D_{+}^{\alpha}(y), D_{+}^{\alpha}(y)=J D_{-}^{\alpha}(y) .
$$

By (3.4), we have

$$
0=\left(\nabla_{X}(A A)\right) Y=A\left(\nabla_{X} A\right) Y+\left(\nabla_{X} A\right) A Y,
$$

from which it follows that if $Y \in D_{+}^{\alpha}(y)$, then

$$
A\left(\nabla_{X} A\right) Y=-\lambda_{\alpha}\left(\nabla_{X} A\right) Y
$$

which means that $\left(\nabla_{X} A\right) Y \in D_{-}^{\alpha}(y)$. Similarly, if $Y \in D_{--}^{\alpha}(y)$, then $\left(\nabla_{X} A\right) Y \in$ $D_{+}^{\alpha}(y)$. Moreover, as is easily seen, if $Y \in D_{+}^{\alpha}(y)$ or $Y \in D_{-}^{\alpha}(y)$, then $(J A) Y \in D_{-}^{\alpha}(y)$ or $(J A) Y \in D_{+}^{\alpha}(y)$, respectively.

Consequently, if $X \in D_{-}^{\alpha}(y)$ and $Y \in D_{+}^{\beta}(y)(\beta=1,2, \cdots, l)$, then from the Codazzi equation

$$
\left(\nabla_{X} A\right) Y-\left(\nabla_{Y} A\right) X-s(X) J A Y+s(Y) J A X=0,
$$

we have

$$
\left(\nabla_{X} A\right) Y=s(X) J A Y \text {. }
$$

Similarly, when $X \in D_{+}^{\alpha}(y)$ and $Y \in D_{-}^{\beta}(y)$, we also have (3.5).

Next, we consider the case where $X \in D_{-}^{\alpha}(y)$ and $Y \in D_{-}^{\beta}(y) . D_{+}^{\beta}(y)=$ $J D_{-}^{\beta}(y)$ means that if $Y \in D_{-}^{\beta}(y)$, then $J Y \in D_{+}^{\beta}(y)$. Therefore since $X \in$ $D_{-}^{\alpha}(y)$ and $J Y \in D_{+}^{\beta}(y)$, by (3.5) we have

$$
\left(\nabla_{X} A\right) J Y=s(X) J A(J Y)=s(X) A Y
$$

or by (2.18)

$$
-J\left(\nabla_{X} A\right) Y=s(X) A Y,
$$

from which we have (3.5).

Similarly, when $X \in D_{+}^{\alpha}(y)$ and $Y \in D_{+}^{\beta}(y)$, we also have (3.5). For the other formula of (3.1), making use of (2.17) and (3.5), we have

$$
\begin{aligned}
\nabla_{X}(J A) Y & =\left(\nabla_{X} J\right) A Y+J\left(\nabla_{X} A\right) Y \\
& =J\left(\nabla_{X} A\right) Y=J s(X) J A Y=-s(X) A Y .
\end{aligned}
$$

Thus, we have $\nabla_{X}^{\prime} \sigma=0$. Consequently, the proof of Theorem is complete.

\section{REFERENCES}

[1] J. ERbacher, Isometric immersions of constant mean curvature and triviality of the normal connection, Nagoya Math. J., 45 (1971), 139-165.

[2] S. Кото̄, Some theorems on almost Kählerian spaces, J. Math. Soc. Japan, 12 (1960), 422-433.

[3] S. Sawaki and K. Sekigawa, Almost Hermitian manifold with constant holomorphic sectional curvature, J. Differential Deometry, 9 (1974), 123-134. 
[4] B. Sмүтн, Differential geometry of complex hypersurfaces, Thesis, Brown University, 1966.

[5] T. TAKahashi, Hypersurfaces with parallel Ricci tensor in a space of constant holomorphic sectional curvature, J. Math. Soc. Japan, 19 (1967), 199-204.

[6] K. Yano and S. IshinARA, Submanifolds with parallel mean curvature vector, J. Differential Geometry, 6 (1971), 95-118.

Department of Mathematics

NiIgATA UNIVERSITY

NiIgATA, 950-21, JAPAN 
